\title{
Malignant Peripheral Nerve Sheath Tumor of the Mandible: A Case Report and Review of Literature
}

\author{
Shyama Prem S ${ }^{\mathrm{a}}$, S Gangothri ${ }^{\mathrm{a}}$, K Sathyanarayana Reddy ${ }^{\mathrm{b}, \mathrm{e}}$, \\ Debdatta Basuc ${ }^{\mathrm{c}}$, Aravind Ramkumard
}

\begin{abstract}
Malignant peripheral nerve sheath tumor of the mandible is an uncommon tumor. We describe a rare case of MPNST involving the mandible with chondrosarcomatous differentiation. It is important to recognize the sarcomatous origin in such tumors and distinguish it from the malignant divergent elements since the management strategy varies considerably. The difficulties faced in MPNST involving the head and neck region is to obtain adequate negative margins with surgery, since the most important prognostic factor for survival is positive margins. The treatment approach is similar to that of other soft tissue sarcomas. Adjuvant radiotherapy plays an important role in decreasing local recurrence.
\end{abstract}

Keywords: Malignant; Nerve sheath; Sarcoma; Heterologous differentiation; Adjuvant; Radiotherapy; Surgical margins; Chemotherapy

\section{Introduction}

Malignant peripheral nerve sheath tumors (MPNSTs) are a rare variety of soft tissue sarcomas which arise from major or minor peripheral nerves and its branches or from the sheaths of peripheral nerve fibers. MPNSTs are mainly located in the buttocks, thighs, brachial plexus, and para-spinal region [1]. Divergent differentiation with heterologous elements, such as bone, cartilage, and glandular tissue, has been reported previously, without clinically significant differences in tumor behavior.

To the best of our knowledge, very few cases of MPNST of the mandible have been reported in literature [2]. We report the clinical presentation and management in a patient with MPNST of the mandible with chondrosarcomatous differentiation, along with a review of the literature.

\section{Case Report}

A 43 year old house-wife presented with a nodular lesion in the left lower alveolus of 3 months duration. She gave a past history of excision and marginal mandibulectomy for a similar swelling one month back in a private hospital which was reported as a benign nerve sheath tumor and she was kept on follow up.

On local examination, there was a firm, non fluctuant lobulated swelling measuring $8 \times 5 \mathrm{~cm}$ in the left lower alveolus with fixity to the underlying mandible. Rest of the oral cavity appeared normal. On general examination, there was no lymphadenopathy, or signs of neurofibromatosis. X-rays

of the mandible showed a lytic lesion in the left mandible

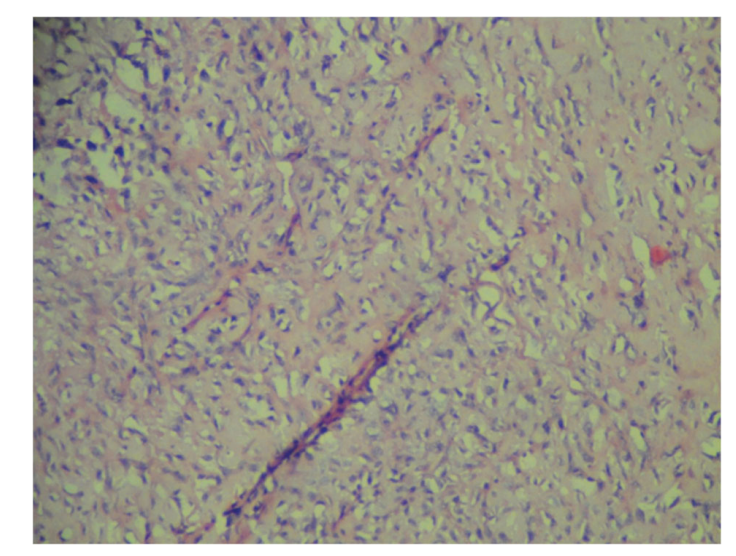

Figure 1: MPNST with malignant spindle cells.

\footnotetext{
${ }^{a}$ Department of Radiotherapy, JIPMER, Puducherry-605006, India

${ }^{\mathrm{b}}$ Regional Cancer Centre and Head of Department, Department of Radiotherapy, JIPMER, Puducherry-605006, India

${ }^{c}$ Department of Pathology, JIPMER, Puducherry-605006, India

${ }^{\mathrm{d}}$ Department of Surgical Oncology, JIPMER, Puducherry-605006, India

${ }^{\mathrm{e}}$ Corresponding author: K Sathyanarayana Reddy.

Email: atsahara11@yahoo.com
}

doi: $10.4021 / \mathrm{jnr} 74 \mathrm{e}$ 


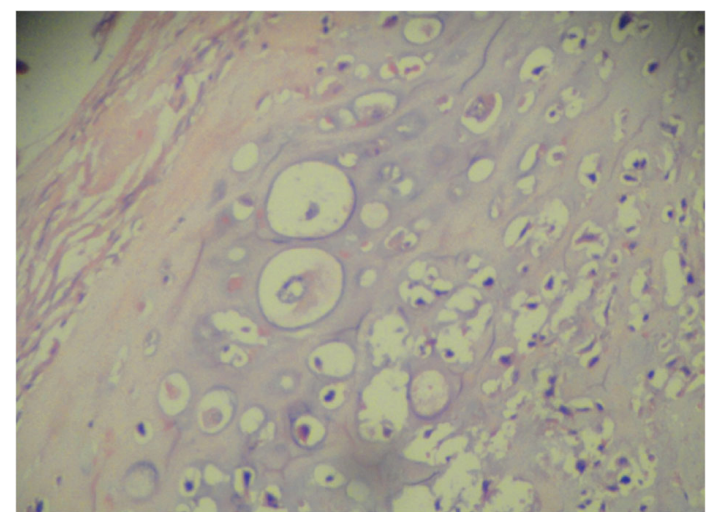

Figure 2: MPNST with chondrosarcomatous differentiation.

with soft tissue component. CT scan revealed an enhancing lesion in the left lower alveolus eroding the underlying mandible without significant lymph node enlargement. Metastatic workup consisting of CT thorax and upper abdomen and bone scans were normal. Tru cut biopsy revealed a peripheral nerve sheath tumor possibly MPNST. In view of the histology of soft tissue sarcoma she underwent wide local excision of the tumor with segmental mandibulectomy and free fibula flap reconstruction. A postoperative wound infection with MRSA was treated successfully with a twenty one day course of vancomycin.

Grossly, the operated specimen revealed a nodular growth with surface ulceration measuring $8.5 \times 7.0 \times 6.5$ $\mathrm{cm}$. The tumor was infiltrating the mandible. Microscopically, the tumor was a high-grade MPNST with high cellularity and brisk mitotic activity (up to 6/10 high-power fields) with foci of necrosis. The tumor was composed of spindle-shaped cells with marked nuclear pleomorphism and giant cells (Fig.1). There was focal malignant cartilaginous differentiation (Fig. 2). The overlying skin was free of tumor. On immune-histochemistry, the tissue was strongly and diffusely positive for Vimentin, S100 and Neuron-specific enolase and negative for Cytokeratin, Factor V111 and CD34. Based on these findings, the patient was diagnosed with an MPNST high grade pleomorphic variant with heterologous chondrosarcomatous differentiation of the mandibular region.

In view of the large size and high grade nature of the tumor the patient was planned for adjuvant radiotherapy (RT). Eight weeks after surgery, once the wound had healed completely, RT was delivered using anterior and lateral portals with 30-degree wedges. A total of $60 \mathrm{~Gy}$ was delivered in 30 fractions at 2.0 Gy per fraction over 6 weeks. A repeat CT 12 months after surgery is normal and she has no evidence of systemic metastasis.

\section{Discussion}

MPNSTs are defined by the World Health Organization as a subcategory of soft tissue sarcomas arising from a peripheral nerve or showing a nerve sheath differentiation, with the exception of tumors originating from the epineurium or the peripheral nerve vasculature.

It can arise de-novo or more often from dedifferentiation within a preexisting neurofibroma in patients with neurofibromatosis type 1 (NF-1) [3]. In around $10 \%$ of the cases MPNST can also arise subsequent to radiation at that site [4]. Since our patient had undergone surgery for a presumed benign solitary neurofibroma, MPNST could have resulted from malignant transformation of an underlying neurofibroma.

Most of MPNSTs occur between 20 and 50 years of age and occurs in equal frequency in both the sexes [1]. Our patient was a 43 year old female and did not have any evidence of NF1.

MPNST typically arise from major nerve trunks like the sciatic nerve, brachial plexus and sacral plexus [1]. Therefore the most common locations for this type of tumor are the extremities and the trunk; head and neck involvement is seen in only $15 \%$ of cases [2]. When head and neck tumors do occur it involves mainly the parotid gland or the infratemporal fossa [4]. MPNST arising in the mandible is very uncommon, and fewer than 50 cases of a maxillomandibular site have been reported in the literature [4].

Magnetic resonance imaging is the investigation of choice because it reveals the nerve origin and the relationship to adjacent structures [5]. Contrast enhanced CT is inferior to MRI in revealing the origin from the nerve but can show the erosion of the mandible and widening of the mandibular canal.

Pathological diagnosis is made by tru-cut biopsy, open incisional biopsy, or excisional biopsy. Fine needle aspiration cytology is inadequate for the assessment of tumor type and grade. Since the clinical differential diagnosis in our patient included a benign nerve sheath tumor and an alveolar carcinoma which are more common and the management strategy differs for all, our patient underwent a tru cut biopsy which was reported as MPNST.

\section{Histology}

Histopathologically, these are highly cellular tumors that characteristically show a fascicular pattern, hyperchromatic spindle cells with elongated nuclei, and abundant cytoplasm. The majority of tumors show geographic necrosis. MPNSTs vary from low grade lesions to the more common high grade tumors which have brisk mitotic activity [6]. In 50 - $70 \%$ of the MPNSTs, scattered tumor cells express S 100 protein [7]. S-100 positivity is not seen in other spindle cell sarcomas. Also, some cases of MPNST stain positive for neuronspecific enolase.

In our case of MPNST the tissue was strongly and diffusely positive for Vimentin, S100 and Neuron-specific 
enolase. It was negative for Cytokeratin, which excluded the possibility of squamous cell carcinoma. MPNSTs occasionally show histological evidence of focal divergent differentiation to rhabdomyosarcoma, osteosarcoma, chondrosarcoma, angiosarcoma, epithelial elements or in combination. Ducatman, et al. noted that the presence of divergent differentiation did not significantly affect the prognosis [1]. In our patient the tumor showed evidence of focal malignant cartilaginous differentiation. Diagnostically, it is important to recognize the sarcomatous origin in such tumors and distinguish it from the malignant divergent elements since the management strategy varies considerably.

\section{Clinical course and treatment}

The optimum management of MPNST still remains unclear. The International Consensus Group has recommended that the current management of MPNST should be identical to that of any other soft tissue tumors [8].

MPNSTs have been reported to be highly aggressive and have a high propensity to metastasize to distant sites [9]. They also tend to recur locally despite aggressive surgical approaches. Large tumor size $(>5 \mathrm{~cm})$, the presence of neurofibromatosis and gross total resection are the most important prognostic factors in MPNST [10]. In the study from the University of Toronto mortality was significantly higher in patients with positive margins compared to those with negative margins [11]. Our patient underwent wide local resection with segmental mandibulectomy with free fibula flap reconstruction which ensured negative margins.

Adjuvant RT is indicated for all high-grade lesions as well as low-grade tumors with positive margins. The supportive literature is generally in the context of sarcomas and not specific to MPNSTs [7]. Since obtaining negative margins is often difficult in the head and neck, adjuvant RT plays a definitive role in MPNST of the head and neck. In a recent retrospective study of 62 patients with MPNSTs, the authors concluded that there was a definite role for postoperative RT (5) [12]. In a study by Basso Ricci adjuvant postoperative RT improved local control [13]. In view of the high grade nature of the tumor and large tumor size both indicators for increased local recurrence, our patient was given radiotherapy to a total dose of $60 \mathrm{~Gy}$.

The role of chemotherapy is usually limited to the treatment of locally advanced and metastatic disease [14]. Carli et al has advocated primary chemotherapy in unresectable tumors to aid tumor shrinkage and a possible macroscopic complete resection [15].

Disease-free survival and overall survival were reported to be approximately $64 \%$ and $30 \%$ at 5 years, respectively. This is in contrast to the 5-year survival rates of 72 to $78 \%$ reported in the soft tissue sarcomas (STS), thereby suggesting the MPNST has a distinctly worse prognosis than other STS. Our patient was treated aggressively and she remains disease free after 2 years.

\section{Conclusion}

MPNST of the mandible is a very rare tumor and the presence of heterologous differentiation does not alter the management. The challenge in a head and neck MPNST is to obtain adequate negative margins with surgery since the most important prognostic factor for survival is negative margins. The overall treatment approach is similar to that of other soft tissue sarcomas. Adjuvant radiotherapy is indicated because of the high incidence of local recurrence.

\section{Grant Support}

None.

\section{Financial Disclosures}

None.

\section{References}

1. Ghosh BC, Ghosh L, Huvos AG, Fortner JG. Malignant schwannoma. A clinicopathologic study. Cancer. 1973;31(1):184-190.

2. Colmenero C, Rivers T, Patron M, Sierra I, Gamallo C. Maxillofacial malignant peripheral nerve sheath tumours. J Craniomaxillofac Surg. 1991;19(1):40-46.

3. Ducatman BS, Scheithauer BW, Piepgras DG, Reiman HM, Ilstrup DM. Malignant peripheral nerve sheath tumors. A clinicopathologic study of 120 cases. Cancer. 1986;57(10):2006-2021.

4. Ducatman BS, Scheithauer BW. Postirradiation neurofibrosarcoma. Cancer. 1983;51(6):1028-1033.

5. Chhabra A, Soldatos T, Durand DJ, Carrino JA, McCarthy EF, Belzberg AJ. The role of magnetic resonance imaging in the diagnostic evaluation of malignant peripheral nerve sheath tumors. Indian J Cancer. 2011;48(3):328-334.

6. Woodruff JM, Perino G. Non-germ-cell or teratomatous malignant tumors showing additional rhabdomyoblastic differentiation, with emphasis on the malignant Triton tumor. Semin Diagn Pathol. 1994;11(1):69-81.

7. Weiss SW, Langloss JM, Enzinger FM. Value of S-100 protein in the diagnosis of soft tissue tumors with particular reference to benign and malignant Schwann cell tumors. Lab Invest. 1983;49(3):299-308.

8. Ferner RE, Gutmann DH. International consensus statement on malignant peripheral nerve sheath tumors in neurofibromatosis. Cancer Res. 2002;62(5):1573-1577. 
9. Collin C, Godbold J, Hajdu S, Brennan M. Localized extremity soft tissue sarcoma: an analysis of factors affecting survival. J Clin Oncol. 1987;5(4):601-612.

10. Wilson AN, Davis A, Bell RS, O'Sullivan B, Catton C, Madadi F, Kandel R, et al. Local control of soft tissue sarcoma of the extremity: the experience of a multidisciplinary sarcoma group with definitive surgery and radiotherapy. Eur J Cancer. 1994;30A(6):746-751.

11. Angelov L, Davis A, O’Sullivan B, Bell R, Guha A. Neurogenic sarcomas: experience at the University of Toronto. Neurosurgery. 1998;43(1):56-64; discussion 64-55.

12. Longhi A, Errani C, Magagnoli G, Alberghini M, Gambarotti M, Mercuri M, Ferrari S. High grade malignant peripheral nerve sheath tumors: outcome of 62 patients with localized disease and review of the literature. J Chemother. 2010;22(6):413-418.

13. Basso-Ricci S. Therapy of malignant schwannomas: usefulness of an integrated radiologic. Surgical therapy. J Neurosurg Sci. 1989;33(3):253-257.

14. Moretti VM, Crawford EA, Staddon AP, Lackman RD, Ogilvie CM. Early outcomes for malignant peripheral nerve sheath tumor treated with chemotherapy. Am J Clin Oncol. 2011;34(4):417-421.

15. Carli M, Ferrari A, Mattke A, Zanetti I, Casanova M, Bisogno G, Cecchetto G, et al. Pediatric malignant peripheral nerve sheath tumor: the Italian and German soft tissue sarcoma cooperative group. J Clin Oncol. 2005;23(33):8422-8430. 